\title{
Predicting the Effective Dose of 5-Aminolevulinic Acid to Protect Humans From Renal Ischemia-Reperfusion Injury: A Study in Micro Miniature Pigs
}

\author{
Kiyotaka Fujine ${ }^{\mathrm{a}, \mathrm{b}}$, Motoaki Sano ${ }^{\mathrm{c}, \mathrm{e}}$, Yoshinori Katsumata ${ }^{\mathrm{c}, \mathrm{d}}$, \\ Kazuki Sato ${ }^{\text {d, Eiji Kobayashi }}{ }^{\mathrm{b}}$
}

\begin{abstract}
Background: In rodent models, 5-aminolevulinic acid (5-ALA) was shown to prevent renal ischemia-reperfusion injury, but the data did not allow estimation of the human equivalent dose (HED). Therefore, this study evaluated the renal effects of 5-ALA and estimated the HED in micro miniature pigs, which have a metabolism closer to that of humans.
\end{abstract}

Methods: We analyzed the efficacy and pharmacokinetics of 5-ALA by administering it to nine micro miniature pigs 2 days before renal ischemia-reperfusion injury. A pathological score was calculated on the basis of renal tissue damage; the blood concentration of 5-ALA required to prevent damage was estimated from the expected pathological score by back-calculating it from the obtained approximate expression. The pharmacokinetics data obtained in phase 1 human trials (5-ALA dose vs. blood concentration curve) were used to calculate the HED.

Results: Dose-dependent improvement in tissue damage (rated as a pathological score) was confirmed 7 days after reperfusion. In each animal, the blood concentrations of 5-ALA correlated with the pathological score. We estimated that doses of approximately $1.6 \mathrm{mg} / \mathrm{kg}$ and $13.4 \mathrm{mg} / \mathrm{kg}$ would be required to obtain histopathology scores of 4 and 3.7 in humans, respectively.

Conclusions: This is the first study to show that the HED of 5-ALA can be calculated from efficacy and pharmacokinetics data obtained in micro miniature pigs.

Manuscript submitted November 1, 2020, accepted November 9, 2020

Published online January 30, 2020

aPharmaceutical Research Department, Neopharma Japan Co., Ltd., Tokyo, Japan

${ }^{b}$ Department of Organ Fabrication, Keio University School of Medicine, Tokyo, Japan

'Department of Cardiology, Keio University School of Medicine, Tokyo, Japan

${ }^{d}$ Institute for Integrated Sports Medicine, Keio University School of Medicine, Tokyo, Japan

${ }^{e}$ Corresponding Author: Motoaki Sano, Department of Cardiology, Keio University School of Medicine, Tokyo, Japan. Email: msano@a8.keio.jp

doi: https://doi.org/10.14740/jcs429
Keywords: 5-aminolevulinic acid; Renal ischemia-reperfusion injury; Pathological scoring; Micro miniature pig; Human equivalent dose

\section{Introduction}

5-aminolevulinic acid (5-ALA) is a natural amino acid found in the mitochondria that is involved in energy production in all living organisms. It is intracellularly converted into protoporphyrin IX (PPIX), which in animals then forms a complex with iron to create heme, a component of the oxygen carrier hemoglobin.

5-ALA has been introduced into health foods with the aim to achieve beneficial effects in several human diseases related to abnormal energy metabolism $[1,2]$; however, the effects of 5-ALA vary depending on its concentration. At low concentrations $(2.5 \mathrm{mg} / \mathrm{kg}), 5$-ALA is effective in activating the electron transport system of mitochondria. Extremely high doses of 5-ALA $(20 \mathrm{mg} / \mathrm{kg})$ are used for photodynamic therapy, which aims to treat cancer through the accumulation of PPIX after administration of 5-ALA [3]. In rodent models, administration of a high dose of 5-ALA (30 - $100 \mathrm{mg} /$ $\mathrm{kg}$ ) has been reported to induce heme oxygenase-1, which can prevent renal ischemia-reperfusion injury [4], have antiinflammatory effects in liver [5] and kidney injuries [6], and induce immune tolerance [7]. Even though 5-ALA has been used in vivo in humans and has extremely low toxicity, further tests in animals that are closely related to humans are required to estimate the human equivalent dose (HED) because the results of animal experiments differ depending on the concentration used [8].

5-ALA was previously shown to prevent renal ischemiareperfusion injury in mouse models. However, micro miniature pigs are better suited for experiments to outline cell therapies and drug usages that can be applied to humans [9]. Furthermore, these pigs are preferable to domestic pigs because their body weight does not exceed $20 \mathrm{~kg}$ even in adulthood. Therefore, this study aimed to verify the HED of 5-ALA in micro miniature pigs by using the known pathological changes after renal ischemia-reperfusion injury in these animals as the evaluation points to convert the concentrations of 5-ALA to 


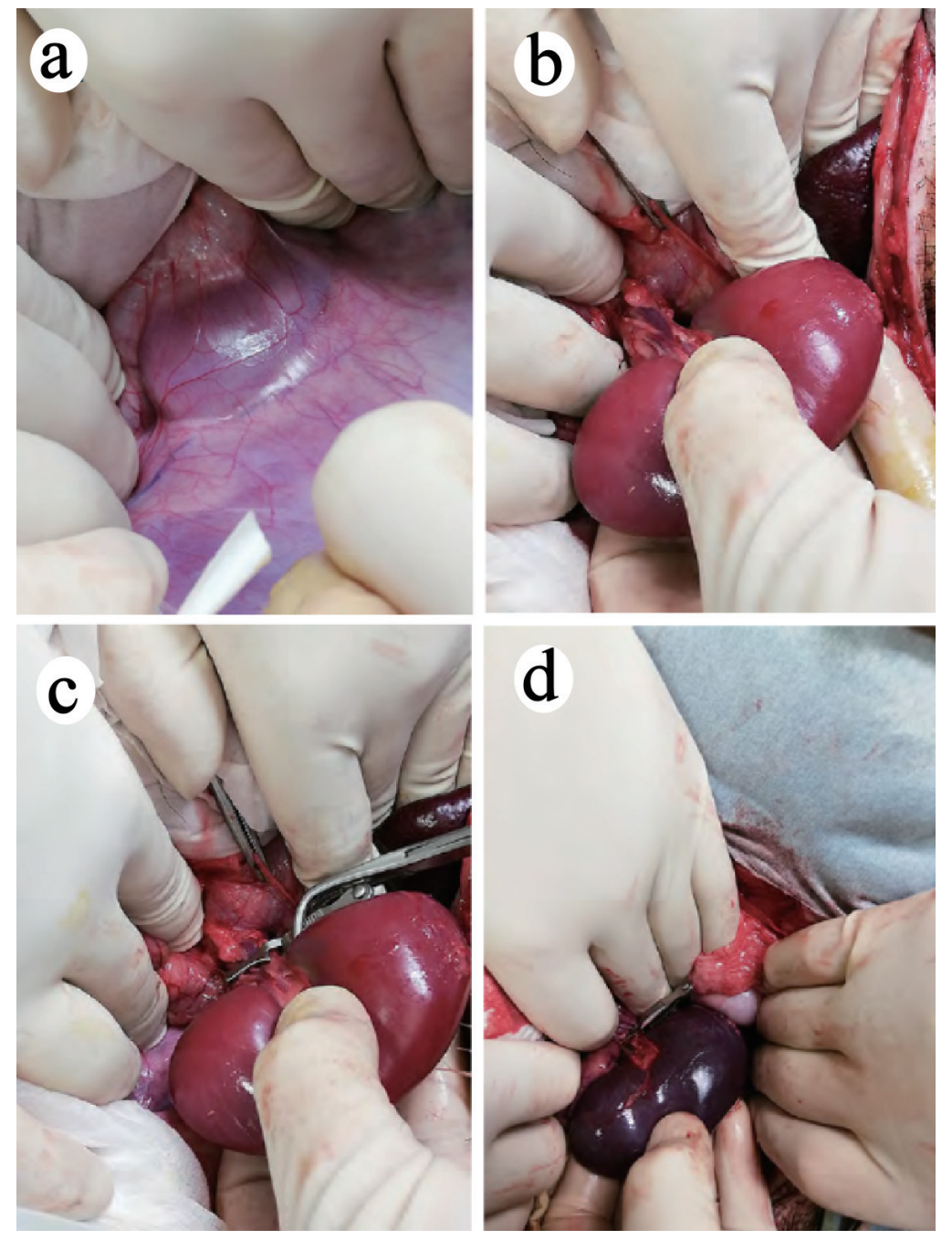

Figure 1. Surgical renal ischemia in the micro miniature pigs. (a, b) The left kidney was exposed and Gerota's fascia was stripped from the left outer side. Renal arteries and veins are visible. (c) A vascular clip was placed on the left renal arteries and veins, and warm ischemia was performed on the left kidney. (d) One hour after inducing ischemia, the clip was removed, and blood reperfusion was confirmed.

the HED. The preventive effects of 5-ALA on renal ischemiareperfusion injury were pathologically verified with two concentrations. This is the first study to verify the pharmacological effects of 5-ALA products with adequate background as health foods on renal ischemia-reperfusion injury in a micro miniature pig model.

\section{Materials and Methods}

\section{Laboratory animals and drugs}

The study used three male and six female 12-month-old mature micro miniature pigs that were born during the same period in the same facility (Fuji Micra Co., Ltd, Shizuoka, Japan). The mean weight of the pigs was $16.2 \pm 0.7 \mathrm{~kg}$. The animals were organized into the following three groups $(n=3$ in each group (one male and two females)): normal food group (control group), $10 \mathrm{mg} / \mathrm{kg} 5$-ALA administration group (5-ALA$\mathrm{HCl} 10 \mathrm{mg} / \mathrm{kg}$ + sodium ferrous citrate (SFC) $7.9 \mathrm{mg} / \mathrm{kg}$ ), and $100 \mathrm{mg} / \mathrm{kg}$ 5-ALA administration group (5-ALA-HCl $100 \mathrm{mg} /$ $\mathrm{kg}+\mathrm{SFC} 78.5 \mathrm{mg} / \mathrm{kg}$ ). The molar ratio of 5-ALA to SFC in both 5-ALA groups was 1:0.25.

The lower dose $(10 \mathrm{mg} / \mathrm{kg} 5$-ALA) was converted from the dose in small animals that was found to induce heme oxygenase-1, anti-inflammation, and immune tolerance. The higher dose $(100 \mathrm{mg} / \mathrm{kg} 5$-ALA) was based on the concentration used for photodynamic detection or photodynamic therapy [3].

The micro miniature pigs were administered 5-ALA + SFC a total of three times, namely 48,24 , and 2 h (day -2 , -1 , and 0 , respectively) before treatment for renal ischemiareperfusion injury. 5-ALA was mixed with a small amount of normal food and SFC to form a dumpling. The animals ingested the whole dumpling before breakfast and received normal food thereafter. 


\section{Ethical issues}

The present study was designed according to the principles of the Animal Research: Reporting of In Vivo Experiments (ARRIVE) guidelines [10]. Experiments were performed in accordance with the institutional guidelines and the Japanese law on the protection and management of animals. The full ethical proposal was approved by the Research Council and Animal Care and Use Committee of Keio University (approval no: 12094, 20005). Surgical procedures were performed by a surgeon with experience of more than 200 clinical transplant operations who is a steering member of the transplantation society and a permanent director of the transplantation society of Japan (EK).

\section{Treatment for renal ischemia-reperfusion injury}

Surgical renal ischemia was performed in the micro miniature pigs by a simplified version of the previously described method $[11,12]$. The pigs were fasted for $12 \mathrm{~h}$ before the procedure, with free access to water. An intramuscular injection of xylazine hydrochloride was given 15 min before the induction of isoflurane anesthesia to immobilize the animals. Laparotomy was performed by making a midline incision on the abdomen. The left kidney was exposed, and Gerota's fascia was stripped from the left outer side so that the renal arteries and veins were visible (Fig. 1a, b). After adding $1 \mathrm{~mL}$ (1,000 units) of heparin to this area, a vascular clip was applied to the left renal arteries and veins to induce warm ischemia of the left kidney (Fig. 1c). Then, the roots of the right renal arteries and veins were ligated with 1-0 silk, the right ureter was ligated, and the right kidney was removed. One hour after induction of ischemia, the clip on the left kidney was removed, blood reperfusion was confirmed (Fig. 1d), and the abdomen was closed.

\section{Postoperative management and blood sampling}

An antibiotic ( $100 \mathrm{mg} / \mathrm{kg}$ body weight of kanamycin intramuscular (IM)) was administered once during the operation, and each animal was kept in an individual cage for 7 days after the operation. Blood was collected from the jugular vein as required, and no restraints, such as tubes, were used.

On day 7 , the animals were sufficiently anesthetized and sacrificed by exsanguination, which led to cardiac arrest. Their kidney was then removed, cut along the long axis and immersed in $10 \%$ formalin for pathological evaluation.

\section{Measurement of 5-ALA concentrations in blood}

Blood was sampled $1.5 \mathrm{~h}$ after 5-ALA administration on day 0 , and serum concentrations of 5-ALA were measured according to the previously reported method, with some modifications [13]. Approximately $40 \mu \mathrm{L}$ of $25 \%$ trichloroacetic acid solution was added to $100 \mu \mathrm{L}$ of serum; the solution was mixed and centrifuged, and $10 \mu \mathrm{L}$ of supernatant was collected. Subsequently, the $10 \mu \mathrm{L}$ of supernatant, $240 \mu \mathrm{L}$ of MilliQ water, 250 $\mu \mathrm{L}$ of $0.2 \mathrm{M}$ acetate buffer ( $\mathrm{pH} 3.8$ ), and $1.25 \mathrm{~mL}$ of solution $\mathrm{A}$ (400 mg sodium chloride) were dissolved in $75 \mathrm{~mL}$ of MilliQ water. Thereafter, $10 \mathrm{~mL}$ of ethanol and $15 \mathrm{~mL}$ of acetylacetone were added and mixed well. A sample solution of 250 $\mu \mathrm{L}$ of solution B was prepared, i.e., $4.5 \mathrm{~mL}$ of formalin was added to an appropriate amount of MilliQ water and diluted to $50 \mathrm{~mL}$ with MilliQ water. This sample solution was heated in boiling water for $15 \mathrm{~min}$ and immediately cooled in ice water. The reaction mixture was next applied to a high-performance liquid chromatography system (Nexera X2 system, RF-20A XS fluorescence detector (Shimadzu) and a ZORBAX Eclipse Plus C18 Rapid Resolution High Throughput column $(1.8 \mu \mathrm{m}$, $4.6 \times 50 \mathrm{~mm}$, Agilent) at $40^{\circ} \mathrm{C}$ ). The sample injection volume was $20 \mu \mathrm{L}$, the mobile phase was a $2.5 \%$ acetic acid-methanol mixture $(6 / 4, \mathrm{v} / \mathrm{v})$, and the flow rate was $1.0 \mathrm{~mL} / \mathrm{min}$.

\section{Pathological evaluation of renal ischemia}

Pathological changes in the kidney excised on day 7 were scored on the basis of the evaluation criteria used in previous mouse models [4]. For histopathological examination, coronal sections were cut, fixed with $10 \%$ formaldehyde and embedded in paraffin. A 5- $\mu \mathrm{m}$ section was prepared and stained with hematoxylin and eosin. Five fields of view each of the cortex and medulla (including the corticomedullary junction; total of 10 fields) were randomly imaged under a magnification of $200 \times$. The incidence rate of renal disorders, including tubular degeneration (such as tubular necrosis), disappearance of the brush border of the proximal tubules, and tubule dilation, was scored as follows: $0-5 \%, 0 ; 5-10 \%, 1 ; 11-25 \%, 2 ; 26-45 \%, 3$; $46-75 \%, 4$; and $75-100 \%, 5$. The scoring was evaluated by two different pathologists in a blinded manner.

\section{Calculation of the HED}

We calculated the approximate expression of the histopathological score (y, a dose-dependent parameter in the pharmacological evaluation of micro miniature pigs) and the approximate expression of 5-ALA (x) in blood $1.5 \mathrm{~h}$ after administration of 5-ALA with the following equation: $y=-0.128$ $\ln (\mathrm{x})+4.2958$ (1). Furthermore, when we plotted the 5-ALA dose (a) and 5-ALA levels in blood (b) $1 \mathrm{~h}$ after administration in humans, using data from a phase 1 study, we approximated the following function: $\mathrm{b}=f(\mathrm{a})$...(2) (unpublished data). Here, we considered the blood 5-ALA concentration $1 \mathrm{~h}$ after administration in humans to be that at $1.5 \mathrm{~h}$ after administration in the micro miniature pigs; in other words, $x=b$. By deriving the inverse functions of equations (1) and (2), the HED (a) could be calculated from the histopathological score (y) via $\mathrm{x}$ and $\mathrm{b}$. For example, if $\mathrm{y}=4, \mathrm{a}=1.6 \mathrm{mg} / \mathrm{kg}$, and if $\mathrm{y}=3.7, \mathrm{a}$ $=13.4 \mathrm{mg} / \mathrm{kg}$.

\section{Statistics}

We used the score in each group to conduct a one-way analysis 
of variance (one-way ANOVA) and Dunnett's multiple comparison test on the control, $10 \mathrm{mg} / \mathrm{kg} 5$-ALA, and $100 \mathrm{mg} / \mathrm{kg}$ 5-ALA groups. The significance level of these tests was 5\%.

\section{Results}

No animal showed abnormalities under general conditions throughout the study period.

\section{Pathological changes in the kidney after renal ischemia}

The histopathological scores of the kidneys are shown in Table 1. In the control group, in the renal proximal tubule epithelial cells at the corticomedullary junction and just below the capsule we observed moderate levels of necrosis due to pyknosis and cytolysis. Furthermore, the proximal tubules without necrosis showed a slight misalignment of cell nuclei, a mild loss of brush border, and a mild-to-moderate dilation of the lumen from the distal renal tubules to the collecting duct. Compared with the controls, the total mean area in which proximal tubular cells underwent necrosis tended to be smaller in the 10 $\mathrm{mg} / \mathrm{kg}$ 5-ALA group and was significantly smaller in the 100 $\mathrm{mg} / \mathrm{kg}$ 5-ALA group (Fig. 2). Based on these observations, the histopathological score was 4.7 for the control group, 4.3 for the $10 \mathrm{mg} / \mathrm{kg} 5$-ALA group, and 3.6 for the $100 \mathrm{mg} / \mathrm{kg} \mathrm{5-ALA}$ group (Table 1). The one-way ANOVA and Dunnett's multiple comparison test showed that the values were significantly lower in both 5-ALA groups than in the controls $(\mathrm{P}<0.05$; Fig. 3a).

In all animals of the three groups, renal tubule degeneration with congestion was observed in multiple lesions, mainly in the intramedullary zone. The level of change was greatest in the control group, followed by the $10 \mathrm{mg} / \mathrm{kg} 5$-ALA group and $100 \mathrm{mg} / \mathrm{kg} 5$-ALA group. Macrophage and lymphocyte infiltration into the interstitium and tubules did not differ significantly between the three groups, although the control group showed a trend for more inflammatory cells. The serum concentrations of 5-ALA were negatively correlated with the histopathological scores $\left(\mathrm{R}^{2}=0.7551\right)$ (Fig. 3b).

\section{Human equivalent dose}

We estimated that doses of approximately $1.6 \mathrm{mg} / \mathrm{kg}$ and 13.4 $\mathrm{mg} / \mathrm{kg}$ were required to obtain the histopathology scores of 4 and 3.7 in humans, respectively (Fig. 4).

\section{Discussion}

5-ALA has long been considered a functional amino acid in animals and plants and has been used in photodynamic therapy for human diseases, particularly cancer $[3,14]$. 5-ALA is multifunctional and has multiple targets, and its effects differ depending on its concentration; consequently, the optimal dose is assumed to vary for each indication. ALA has only been tested

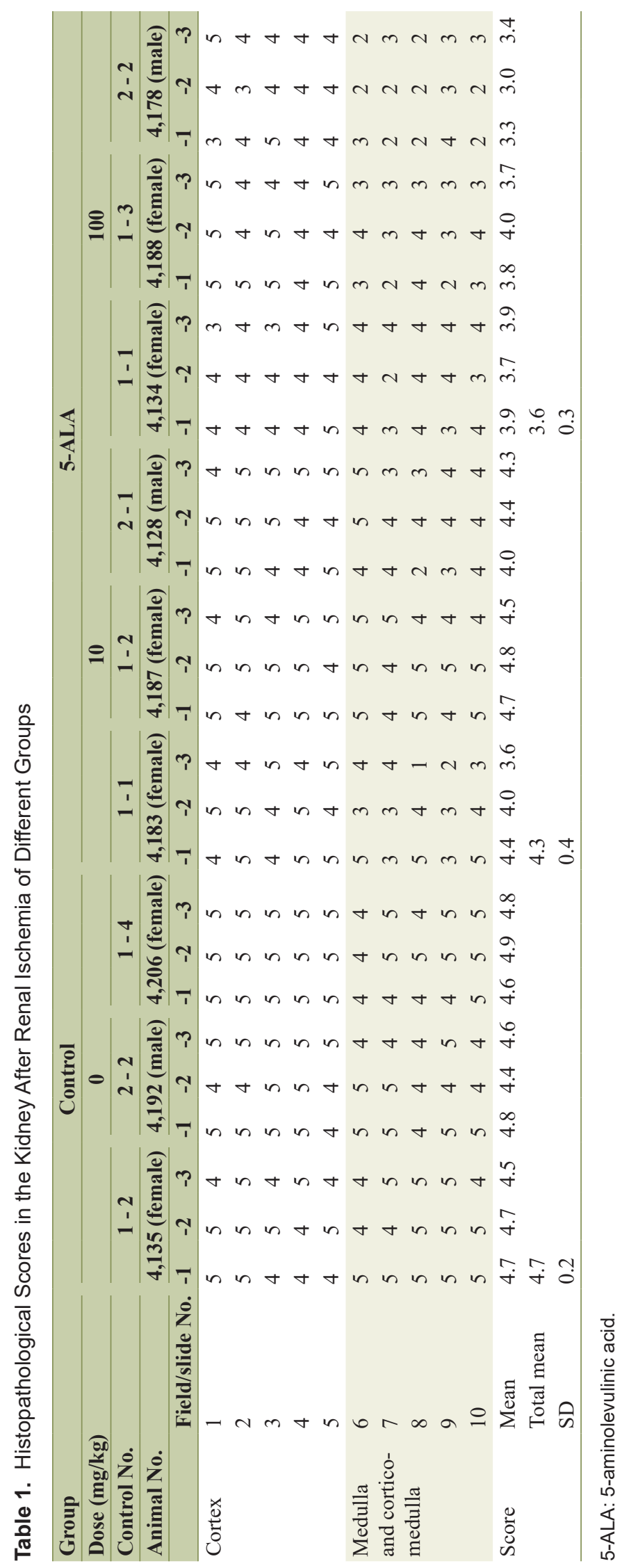




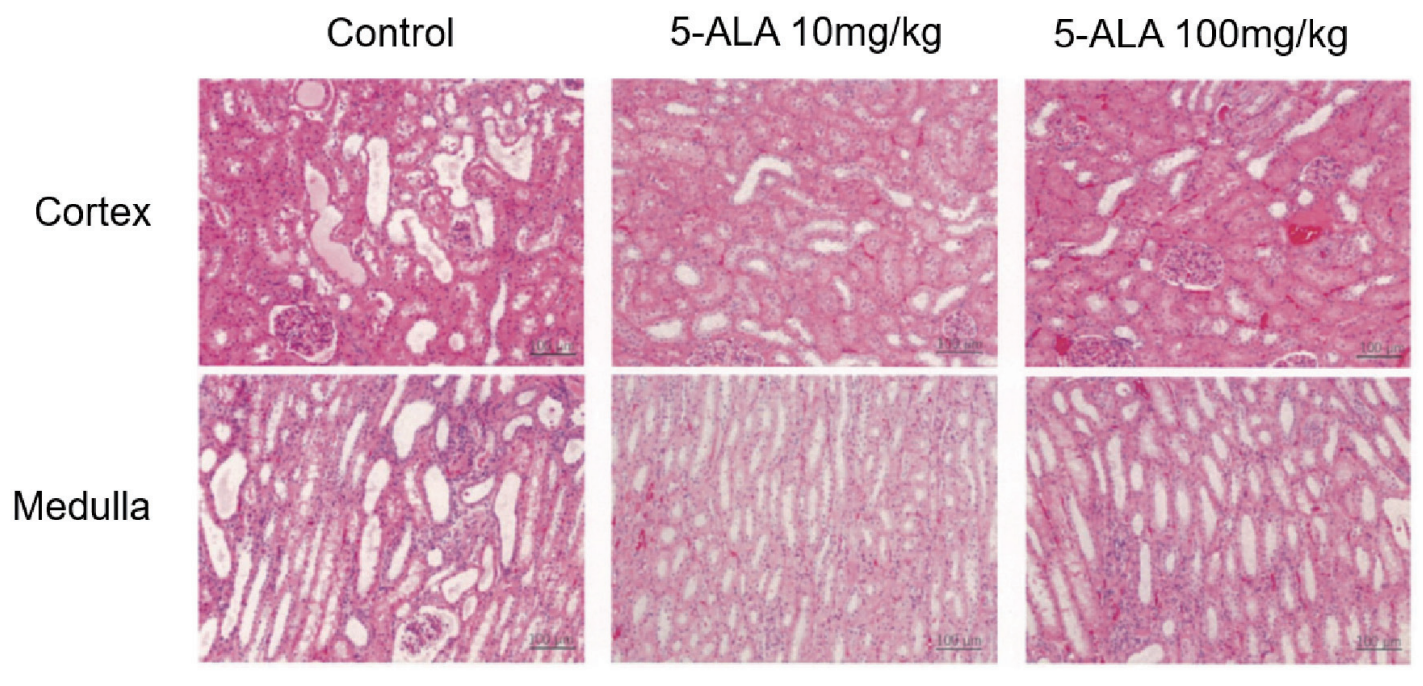

Figure 2. Pathological evaluation of renal ischemia from five fields of view each of the cortex and medulla (including the corticomedullary junction), for a total of 10 fields. The fields of view were imaged randomly under a magnification of $200 \times$ and scored according to the incidence rate of pathological changes, as described in the main text.

in rodents to date, and these studies did not measure pharmacokinetics associated with pharmacodynamics. In vitro studies assumed that 5-ALA absorption levels are different in rodents, pigs, and humans and that human intestinal cells absorb only $8 \%$ of the administered 5-ALA [15]. This difference makes it difficult to predict clinical doses for humans on the basis of in vivo rodent findings. A phase 1 study of the pharmacokinetics of oral 5-ALA showed that the peak serum concentration occurred 1 to $2 \mathrm{~h}$ after administration of $50 \mathrm{mg}$ to $1,500 \mathrm{mg}$ (unpublished data).

Rodents are known to generally rapidly metabolize drugs, and the effective dose of drugs in rodents differs greatly from that in humans. On the other hand, the metabolism of pigs is

\section{a}

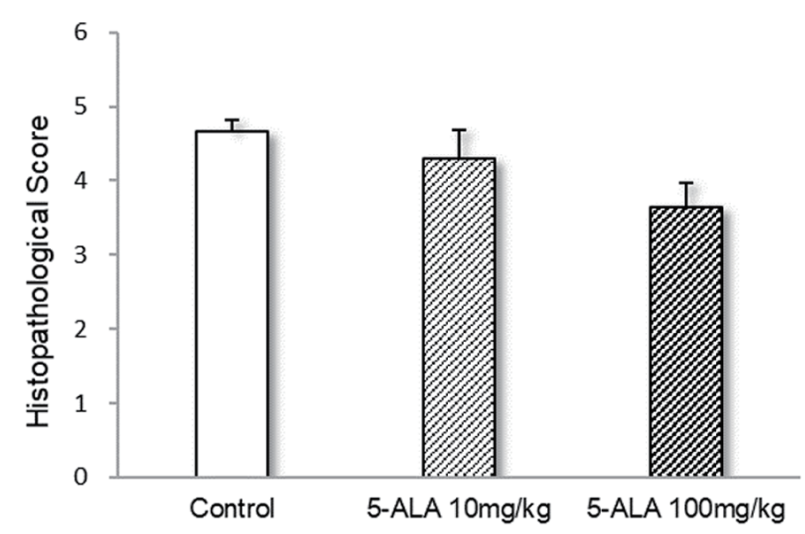

closer to that of humans than rodents. Therefore, evaluating drug metabolism in pigs allows clinical doses in humans to be predicted more accurately. However, domestic pigs grow rapidly, so it is difficult to match their age with humans. Micro miniature pigs are more useful for such studies because they grow more slowly and have a low body weight, which allows experiments to be performed with lower doses of test drugs $[9,16]$. Therefore, in this study we used micro miniature pigs as a renal ischemia-reperfusion injury model to more accurately determine the effective HED and blood concentration of 5-ALA by comparing the pharmacokinetic data on 5-ALA with those in humans.

This is the first report to demonstrate that the clinically

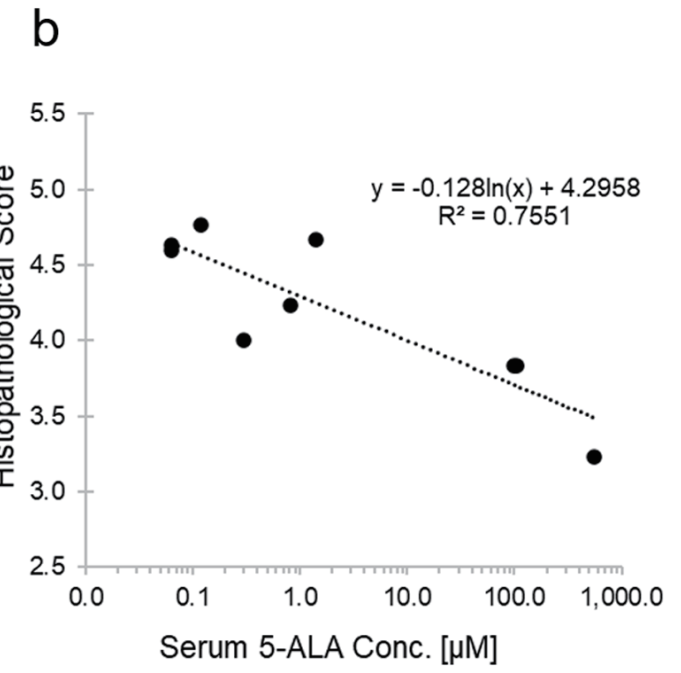

Figure 3. (a) Values represent the mean histopathological scores \pm SD for nine aspects in each group. One-way analysis of variance revealed a statistically significant difference $(P<0.001)$. ${ }^{*}$ Indicates significant difference vs. control in Dunnett's multiple comparison test $(\mathrm{P}<0.05)$. (b) Correlation between histopathological sores and serum 5-ALA concentration. SD: standard deviation; ALA: aminolevulinic acid. 
a

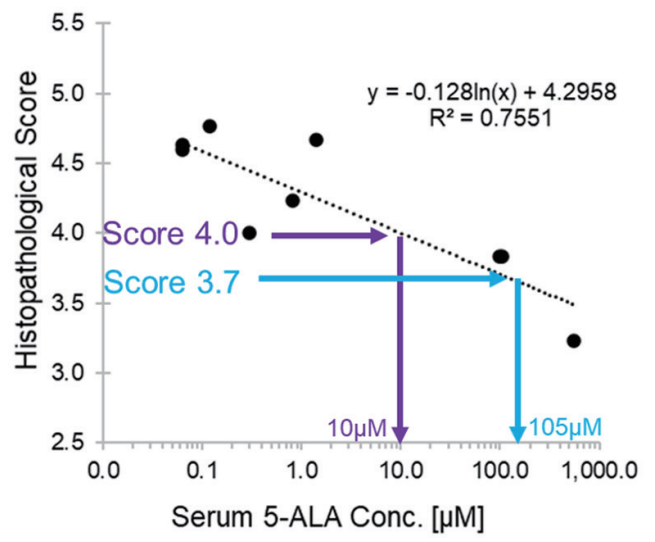

b

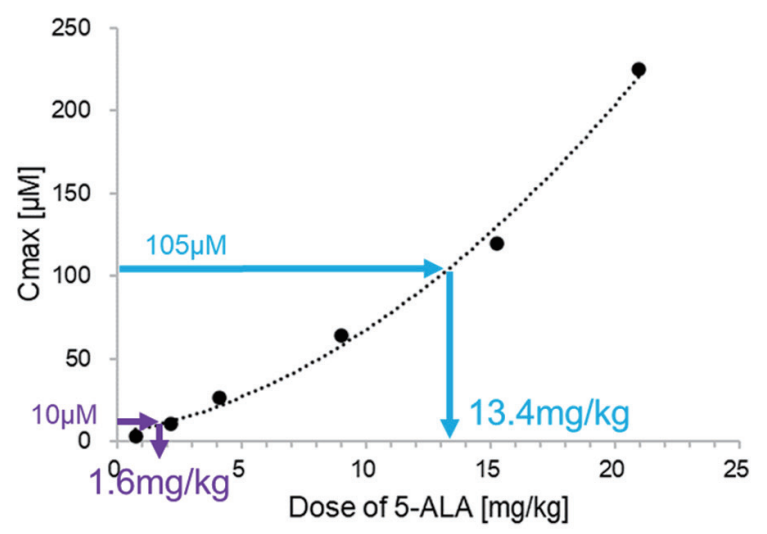

Figure 4. (a) Histopathological score vs. maximum drug concentration (Cmax) of 5-ALA in micro miniature pigs. Approximate curve was used to assume the required concentration from the expected drug efficacy index (histopathological score). (b) Cmax vs. dose of 5-ALA in humans. The approximate curve was used to calculate the required dose of 5-ALA from the assumed blood concentration in (a). Thus, by defining the drug efficacy parameters that correlated with blood concentrations of 5-ALA and obtaining an approximate expression in micro miniature pigs, we were able to estimate the dose of 5-ALA required for humans from the expected index of drug efficacy. 5-ALA: 5-aminolevulinic acid.

effective dose of 5-ALA for humans can be predicted from experiments in animals. The study used micro miniature pigs to verify the inhibitory effect of 5-ALA on renal ischemia-reperfusion injury, which had been proven previously in smaller animals. It also analyzed the efficacy and pharmacokinetics of 5-ALA in each individual animal.

A limitation of the study is that 5-ALA is pharmacologically active on various heme-binding proteins, so the concentration of 5-ALA required for a specific pharmacological activity may differ depending on the desired effect.

The methods used in this study can be used in future studies to estimate the clinically effective dose of 5-ALA in humans by first determining the concentration in an animal model.

\section{Acknowledgments}

This study was conducted according to the recommendations of Motowo Nakajima and Tohru Tanaka (SBI Pharmaceuticals Co., Ltd., Tokyo, Japan). Furthermore, blood concentrations of 5-ALA were measured by Masato Usami (Neophama Japan Co., Ltd., Tokyo, Japan). We would like to express our sincere gratitude to them.

\section{Financial Disclosure}

The study was supported by a research grant from Neopharma Japan Co., Ltd.

\section{Conflict of Interest}

$\mathrm{KF}$ is an employee of Neopharma Japan Co., Ltd., and a visit- ing researcher at Keio University. The remaining authors have no conflicts of interest to declare.

\section{Informed Consent}

Not applicable.

\section{Author Contributions}

EK designed and performed the study. KF, YK, EK, and MS drafted the manuscript and did critical editing. KF and EK assisted and supported in sample collection and subsequent analysis with statistics. YK and KS carefully supervised this manuscript preparation and writing.

\section{Data Availability}

The data supporting the findings of this study are available from the corresponding author upon reasonable request.

\section{References}

1. Rehani PR, Iftikhar H, Nakajima M, Tanaka T, Jabbar Z, Rehani RN. Safety and Mode of Action of Diabetes Medications in comparison with 5-Aminolevulinic Acid (5-ALA). J Diabetes Res. 2019;2019:4267357.

2. Suzuki H, Masuki S, Morikawa A, Ogawa Y, Kamijo YI, Takahashi K, Nakajima M, et al. Effects of 5-aminolevulinic acid supplementation on home-based walking training achievement in middle-aged depressive women: 
randomized, double-blind, crossover pilot study. Sci Rep. 2018;8(1):7151.

3. Stummer W, Pichlmeier U, Meinel T, Wiestler OD, Zanella F, Reulen HJ, ALA-Glioma Study Group. Fluorescence-guided surgery with 5-aminolevulinic acid for resection of malignant glioma: a randomised controlled multicentre phase III trial. Lancet Oncol. 2006;7(5):392401.

4. Hou J, Cai S, Kitajima Y, Fujino M, Ito H, Takahashi K, Abe F, et al. 5-Aminolevulinic acid combined with ferrous iron induces carbon monoxide generation in mouse kidneys and protects from renal ischemia-reperfusion injury. Am J Physiol Renal Physiol. 2013;305(8):F11491157.

5. Li S, Takahara T, Li XK, Fujino M, Sugiyama T, Tsukada $\mathrm{K}$, Liu C, et al. 5-Aminolevulinic acid combined with ferrous iron ameliorate ischemia-reperfusion injury in the mouse fatty liver model. Biochem Biophys Res Commun. 2016;470(4):900-906.

6. Uchida A, Kidokoro K, Sogawa Y, Itano S, Nagasu H, Satoh M, Sasaki T, et al. 5-Aminolevulinic acid exerts renoprotective effect via Nrf2 activation in murine rhabdomyolysis-induced acute kidney injury. Nephrology (Carlton). 2019;24(1):28-38.

7. Hou J, Zhang Q, Fujino M, Cai S, Ito H, Takahashi K, Abe F, et al. 5-Aminolevulinic acid with ferrous iron induces permanent cardiac allograft acceptance in mice via induction of regulatory cells. J Heart Lung Transplant. 2015;34(2):254-263.

8. Nair AB, Jacob S. A simple practice guide for dose conversion between animals and human. J Basic Clin Pharm. 2016;7(2):27-31.

9. Tohyama S, Kobayashi E. Age-appropriateness of por- cine models used for cell transplantation. Cell Transplant. 2019;28(2):224-228.

10. Miura K, Sahara H, Sekijima M, Kawai A, Waki S, Nishimura H, Setoyama K, et al. Protective effect of neutralization of the extracellular high-mobility group box 1 on renal ischemia-reperfusion injury in miniature swine. Transplantation. 2014;98(9):937-943.

11. Kobayashi E, Sano M. Organ preservation solution containing dissolved hydrogen gas from a hydrogenabsorbing alloy canister improves function of transplanted ischemic kidneys in miniature pigs. PLoS One. 2019;14(10):e0222863.

12. Kilkenny C, Browne WJ, Cuthill IC, Emerson M, Altman DG. Improving bioscience research reporting: the ARRIVE guidelines for reporting animal research. PLoS Biol. 2010;8(6):e1000412.

13. Endo Y, Okayama A, Endo G, Ueda T, Nakazono N, Horiguchi $\mathrm{S}$. Improvement of urinary delta-aminolevulinic acid determination by HPLC and fluorescence detection using condensing reaction with acetylacetone and formaldehyde. Sangyo Igaku. 1994;36(2):49-56.

14. Jansen MHE, Kessels J, Nelemans PJ, Kouloubis N, Arits A, van Pelt HPA, Quaedvlieg PJF, et al. Randomized trial of four treatment approaches for actinic keratosis. N Engl J Med. 2019;380(10):935-946.

15. Saito K, Fujiwara T, Ota U, Hatta S, Ichikawa S, Kobayashi M, Okitsu Y, et al. Dynamics of absorption, metabolism, and excretion of 5-aminolevulinic acid in human intestinal Caco-2 cells. Biochem Biophys Rep. 2017;11:105-111.

16. Kobayashi E, Hanazono Y, Kunita S. Swine used in the medical university: overview of 20 years of experience. Exp Anim. 2018;67(1):7-13. 